\title{
Revisión sistemática de evaluaciones económicas en salud para el tratamiento de la obesidad en adultos, 2009-2019
}

\author{
Julie May Sánchez Gómez , María Isabel Betancur Mejía y Jaiberth Antonio Cardona-Arias²
}

Forma de citar Sánchez Gómez JM, Betancur Mejía MI, Cardona-Arias JA. Revisión sistemática de evaluaciones económicas en salud para el tratamiento de la obesidad en adultos, 2009-2019. Rev Panam Salud Publica. 2020;44:e158. https://doi.org/10.26633/ RPSP.2020.158

RESUMEN Objetivo. Analizar las evaluaciones económicas en salud para el tratamiento de la obesidad en adultos publicadas en la literatura mundial entre 2009-2019.

Métodos. Revisión sistemática aplicando las guías Cochrane, PRISMA y CHEERS para estudios de economía de la salud. Se garantizó la exhaustividad, reproducibilidad y calidad metodológica del protocolo de selección y extracción de la información. Se realizó síntesis cualitativa mediante frecuencia por tipo de evaluación económica.

Resultados. Se tamizaron 4493 artículos y sólo 95 cumplieron con los criterios del protocolo, la mayoría de Estados Unidos $(n=38)$, principalmente con estudios de costo-utilidad $(n=39)$ y descripción de costos $(n=18)$. Se incluyeron personas con múltiples comorbilidades, la más frecuente de las cuales fue la diabetes. Las intervenciones más evaluadas fueron las quirúrgicas $(n=53)$, y los desenlaces más frecuentes fueron la reducción de peso y los años de vida ajustados por calidad. La perspectiva de evaluación más frecuente fue la del tercer pagador $(n=46)$ y el horizonte temporal más empleado fue el corto plazo para las intervenciones dirigidas a los estilos de vida y de largo plazo para las quirúrgicas.

Conclusión. Los estudios microeconómicos para el tratamiento de la obesidad se han incrementado en los últimos años; sin embargo, persisten vacíos como la baja aplicación de intervenciones no quirúrgicas, evaluaciones parciales o de costo-efectividad y análisis desde la perspectiva social. Estos constituyen ejes importantes para las prácticas en salud basadas en evidencia y la toma de decisiones relacionando los beneficios en salud con los costos implicados.

Palabras clave Obesidad; control de enfermedades transmisibles; economía de la salud; revisión sistemática; adulto.

La obesidad es un desequilibrio energético con acumulación de grasa corporal, causada por factores genéticos, comportamentales, económicos y sociales ${ }^{1}$. Dada su tendencia creciente desde 1980, constituye una prioridad para la salud pública mundial, con una prevalencia en adultos que oscila desde $4,2 \%$ en Japón hasta $40 \%$ en Estados Unidos ${ }^{2,3}$. Sus consecuencias clínicas incluyen hipertensión arterial, dislipidemias, diabetes, enfermedades coronarias, osteoartritis, problemas respiratorios, apnea del sueño y cánceres ${ }^{4,5}$.

A los efectos clínicos de la obesidad se suman sus graves impactos socioeconómicos, como el incremento del riesgo de muerte prematura, el ausentismo laboral, el riesgo de desempleo y los gastos en salud, al tiempo que se reducen el rendimiento escolar, los ingresos laborales, la productividad, el

Universidad CES, Medellín, Colombia

2 Universidad de Antioquia, Medellín, Colombia $₫$ Jaiberth Antonio CardonaArias, jaiberth.cardona@udea.edu.co. 
producto interno bruto (PIB) y el presupuesto sanitario por el alto costos de las enfermedades crónicas relacionadas ${ }^{6}$.

Tradicionalmente se han aplicado dos enfoques para intervenir sobre la obesidad, el preventivo y el terapéutico. Aunque la prevención es más eficiente, ya que puede evitar las alteraciones asociadas con esta afección e incurrir en menores costos, el enfoque predominante ha sido el terapéutico. Este comprende alternativas centradas en los estilos de vida, que buscan cambios en los comportamientos relacionados con la ingesta calórica y el nivel de actividad física; abordajes farmacológicos, con efectos sobre la absorción o utilización de nutrientes en el cuerpo; y procedimientos quirúrgicos que limitan la capacidad de ingesta y absorción de nutrientes ${ }^{7}$.

Estas intervenciones presentan una amplia diversidad de costos, grado de efectividad, resultados a corto y largo plazo, e impactos en la calidad de vida, entre otros ${ }^{8}$. Tal diversidad puede caracterizarse de manera adecuada mediante una revisión sistemática bajo el enfoque amplio de Cochrane, aplicado a evaluaciones económicas en salud (EES) completas y parciales. Las primeras permiten comparar la efectividad y los costos de al menos dos intervenciones, mientras que las parciales pueden abordar estos componentes (costos y efectos en salud) de manera independiente. Las EES buscan ofrecer información útil para los tomadores de decisiones en diferentes niveles (político, económico, asistencial) para tratar el fenómeno de la obesidad debido a que ofrecen recomendaciones claras sobre la eficiencia en el uso de los recursos sanitarios y las mejores alternativas para la toma de decisiones ${ }^{8}$.

De esta manera, en las últimas décadas a nivel mundial se ha presentado un aumento de este tipo de estudios. A pesar del esfuerzo para mejorar su uniformidad, calidad y la generación de directrices que regulen la presentación y evalúen su calidad, persiste una alta variabilidad metodológica y de resultados en los diferentes países, así como una baja disponibilidad de estudios que sistematicen la evidencia disponible.

El objetivo de esta investigación fue analizar las EES para el tratamiento de la obesidad en adultos publicadas en la literatura mundial entre 2009-2019 mediante una revisión sistemática bajo el enfoque amplio de Cochrane. Este permite una aproximación práctica para sintetizar la producción científica en el tema, sintetizar la evidencia disponible, proponer o consolidar hipótesis, reconocer áreas relevantes para orientar estudios posteriores, y localizar países con necesidad de mayor investigación, entre otros usos ${ }^{9-11}$.

\section{MATERIALES Y MÉTODOS}

\section{Tipo de estudio}

Revisión sistemática de evaluaciones económicas parciales (descripción de costos, análisis de costos, descripción de costos y resultados) y completas (costo-minimización, costoefectividad, costo-utilidad y costo-beneficio) ${ }^{12}$.

\section{Pregunta PICORT: Población, Intervención, Comparador, Desenlaces, Recursos y Horizonte temporal}

Población: adultos con diagnóstico nutricional de obesidad según el índice de masa corporal (IMC).
Intervenciones: cualquier tipo de intervención cuyo objetivo fue la reducción de peso, como un tratamiento farmacológico en el que se describa la dosis y el tiempo; un tratamiento con cualquier técnica quirúrgica bariátrica, como banda gástrica, derivación gástrica en $\mathrm{Y}$ de Roux y gastrectomía en manga; un tratamiento relacionado con los estilos de vida en el que se buscaron cambios en el consumo de alimentos, el nivel de actividad física o cambios conductuales dirigidos a intervenir sobre la obesidad.

Comparador: no se hizo ninguna restricción; puede ser una intervención farmacológica, quirúrgica, de estilos de vida, tratamiento convencional, no tratar $\mathrm{u}$ otros enfoques usados en los estudios sistematizados. En síntesis, los registrados en cada estudio sistematizado.

Desenlaces: no se hizo ninguna restricción. Estas medidas pueden ser pérdida de peso porcentual o absoluto, cambios en el IMC, reducción de la circunferencia de cintura, años de vida ganados, o años de vida ajustados por calidad.

Recursos: costo de la intervención, entendido como el valor de los recursos utilizados por prestar un servicio o realizar una intervención, de acuerdo a la perspectiva tomada en el estudio, con denominación de tipo de moneda y año corriente.

Horizonte temporal: el reportado en cada estudio.

\section{Protocolo de búsqueda y selección de los estudios}

La búsqueda de los estudios se realizó en bases de datos electrónicas de contenido multidisciplinar, inclusive PubMed, Cochrane, Scielo, ScienceDirect, la base de datos especializada NHS Economic Evaluation Database y Google Académico. Los términos para estructurar la estrategia de búsqueda incluyeron palabras claves MeSH y libres. Se emplearon los términos en español obesidad, costo, costo efectividad, costo utilidad, costo beneficio, costo minimización, costo análisis, evaluación económica. Los términos empleados en inglés fueron obesity, cost effectiveness, cost benefit, cost minimization, cost utility, cost, economic evaluation (Las 44 ecuaciones de búsqueda empleadas están disponibles para el lector interesado a través del autor de correspondencia).

Los resultados de las estrategias de búsqueda se administraron en una fuente común (Zotero) para eliminar los duplicados. Se descartaron los artículos que no cumplieron los criterios de inclusión relacionados con la pregunta PICORT, y se excluyeron también los estudios en mujeres gestantes, intervenciones basadas en programas preventivos de la obesidad o con tratamiento adicional para otras enfermedades (especialmente osteomusculares), revisiones sistemáticas, reportes de caso, libros, conferencias y los artículos no disponibles en texto completo.

\section{Evaluación de la reproducibilidad y de la calidad metodológica}

Durante todas las fases, dos investigadores aplicaron las estrategias de búsqueda, criterios de inclusión y exclusión, evaluación de sesgo, obtención de datos. En caso de alguna discrepancia, los dos investigadores intentaron llegar a un consenso y, si este no se lograba, se refería al tercer investigador. Además, la calidad metodológica de los estudios se evaluó mediante la aplicación de la guía Consolidated Health Economic Reporting Standards (CHEERS). 


\section{Análisis de la información}

Para la identificación de los diferentes tipos de EES se consideró la clasificación propuesta por Drummond ${ }^{12}$, y se extrajo autor, año, país, modelo y perspectiva de análisis, comorbilidades, tipo de intervenciones y comparadores, medidas de efectividad y características de los costos. Estos se transformaron a los precios constantes del dólar estadounidense de 2019 a través del índice de precios al consumidor y la tasa de cambio reportada en la Organización para la Cooperación y el Desarrollo Económicos (OCDE) $)^{13}$. Las variables del estudio se describieron con frecuencias absolutas y relativas, se determinó el porcentaje de criterios de la guía CHEERS que cumplió cada estudio y los desenlaces económicos se describieron en términos de costo o costo-utilidad media de cada intervención investigada (en los casos en que se evaluó la misma intervención en varios estudios se reportó el rango).

\section{RESULTADOS}

Se identificaron 4493 estudios, de los cuales sólo 95 cumplieron todos los criterios del protocolo de búsqueda y selección (Figura 1) (la lista completa de artículos incluidos está disponible para el lector interesado a través del autor de correspondencia). La calidad global de los estudios fue moderada, dada la aplicación parcial de la mayoría de ítems, siendo menor la descripción de las fuentes de financiación y la caracterización de la heterogeneidad (Figura 2). Los países con mayor número de evaluaciones económicas fueron Estados Unidos $(40 \%)$ y el Reino Unido (16\%), ambos con una mayor proporción de estudios de costo-utilidad (Figura 3).

Las evaluaciones más frecuentes fueron de costo-utilidad (41\%), seguidas por descripción de costos $(15,8 \%)$, descripción de costos y resultados $(16,8 \%)$, análisis de costo $(17,9 \%)$,

FIGURA 1. Flujograma de búsqueda y selección de estudios
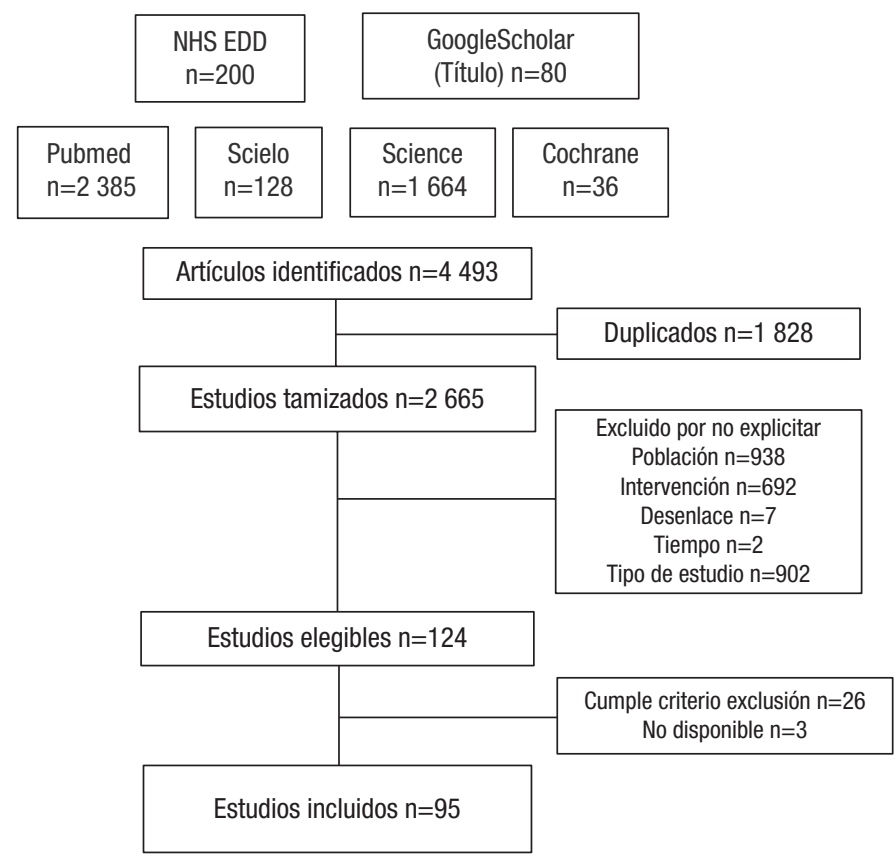

Fuente: Elaboración propia a partir de datos publicados. costo-efectividad $(7,4 \%)$ y un solo estudio de costo-beneficio. El $57 \%$ de los estudios se basó en datos de pacientes y el $43 \%$ en modelaciones; en este último grupo $56 \%$ fueron descriptivas, 29\% modelos Márkov y 15\% simulación de eventos discretos.

La edad de los pacientes osciló entre 18 y 50 años, y fueron más frecuentes los estudios con personas entre los 20-35 años; sólo el 13\% se concentró en obesidad grave y mórbida. Las comorbilidades más frecuentes fueron la diabetes tipo $2(70 \%)$ y las enfermedades cardiovasculares (59\%). Las principales intervenciones fueron las quirúrgicas $(56 \%$ ) y las dirigidas a estilos de vida (34\%); el comparador más usado fue el manejo convencional $(35 \%)$, y las medidas de efectividad comunes fueron la pérdida de peso (14,7\%) y los AVAC (años de vida ajustados por calidad, QALY en inglés) (44\%) cuyo ajuste por calidad se basó en reportes de la literatura y sólo en seis estudios fue realizado con datos del SF-36, SF-12V, EQ-5D y IWQOL-lite aplicado a pacientes. En cuanto a los costos, 79\% usó los costos directos con su estimación de promedios (insumos, medicamentos y personal asistencial) y se encontró una perspectiva enfocada en un tercer pagador en $48 \%$ de los estudios, en el prestador en $35 \%$ y social en $17 \%$ (Cuadroabla 1 ).

Los estudios de descripción de costos se realizaron principalmente para tratamientos quirúrgicos; la estimación de costo fue de corto plazo y principalmente para costos directos asociados con hospitalización y medicamentos. En los estudios de descripción de costo-resultado las medidas más utilizadas fueron el peso y los años de vida ajustados por discapacidad o calidad; la mayoría de las intervenciones estaban asociadas a los estilos de vida (dieta y actividad física en ambientes hospitalario, comunitario o ambulatorio) a corto plazo. En los análisis de costos la principal intervención evaluada fue la quirúrgica; todas se basaron en datos clínicos a corto plazo, desde la perspectiva institucional, y principalmente con costos directos como personal médico, medicamentos, insumos y hospitalización. En las evaluaciones económicas completas predominaron estas mismas características (Tabla 1).

Con base en los costos reportados en las evaluaciones económicas parciales, las mejores intervenciones son las basadas en laparoscopia, mientras que las educativas o conductuales son las más costosas. Sin embargo, al considerar los resultados en salud los programas educativos presentan bajos costos por AVAC ganado, al igual que las intervenciones farmacológicas para los AVAD (años de vida ajustados por discapacidad) y las intervenciones farmacológicas o dirigidas al estilo de vida para la reducción del peso (Tabla 2).

En los análisis de costo-utilidad se reportaron razones incrementales desde USD -6 028, que refleja una intervención dominante con ahorros para el sistema de salud, hasta USD 66097 por AVAC adicional, con una alta diversidad de valores según el tipo de intervención y comparador. De manera general, las razones incrementales demuestran que la intervenciones identificadas, según los umbrales de la Organización Mundial de la Salud, son costo-útiles al estar por debajo de los 3 PIB per cápita de los países en que se realizaron las investigaciones.

\section{DISCUSIÓN}

Los resultados de la revisión sistemática de EES con intervenciones sobre la obesidad mostraron que Estados Unidos ha sido el país que más ha desarrollado este tipo de estudios. Según datos de la OCDE, ${ }^{2}$ este es el país con mayores problemas de 
FIGURA 2. Evaluación de la calidad según criterios de la guía CHEERS

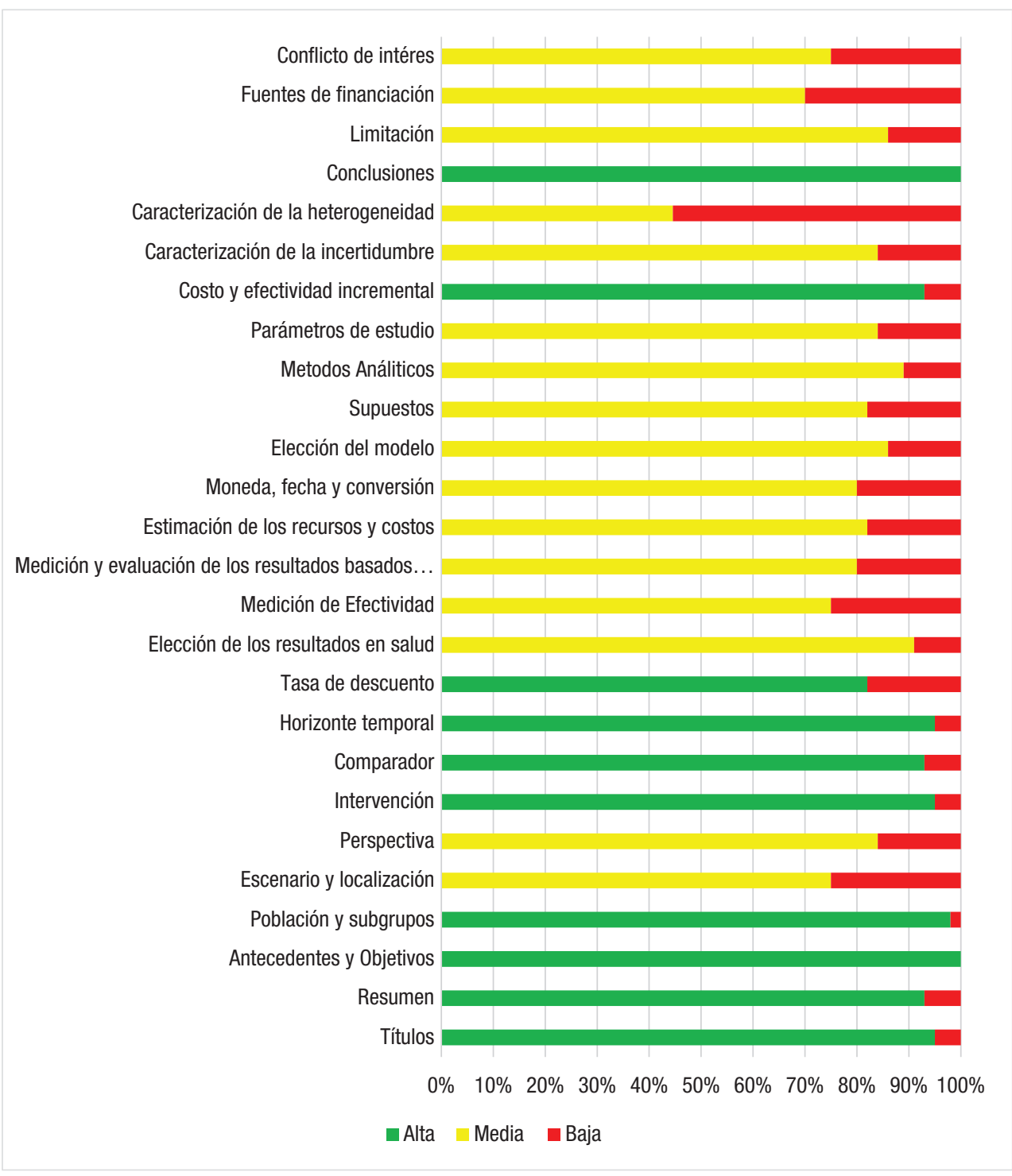

Fuente: Elaboración propia a partir de datos publicados.

obesidad; casi el $40 \%$ de la población adulta la padece, y se necesita información para la toma de decisiones sobre las alternativas disponibles y más eficientes para intervenir sobre la obesidad.

El principal tipo de EES fue el de costo-utilidad, lo cual es relevante en términos de salud pública dado que este tipo de estudio tiene la capacidad de incluir más información sobre la cantidad (años de vida ganados) y calidad de vida; además, generan una métrica comparable entre diferentes condiciones de salud ${ }^{14}$. Pese a esta ventaja, en esta revisión no fue posible identificar la intervención que presentaba la mejor razón de costo-utilidad dada la diversidad de comparadores, aunque todos los tratamientos analizados mediante este tipo de EES permiten evidenciar su alto valor económico y sanitario al implicar inversiones menores a 3 PIB per cápita por AVAC adicional ${ }^{15,16}$.

También es importante destacar que esta revisión incluyó un número importante de estudios de descripción de costos, descripción de costo-resultado y análisis de costos. Una característica importante compartida por la mayoría ellos es que eran EES derivadas de estudios clínicos, a diferencia de las evaluaciones económicas completas que eran diseñadas a partir de los modelos propuestos, en su mayoría cadenas de Márkov. Alsumali et $\mathrm{al}^{17}$ sugieren que los modelos de microsimulación o simulación de eventos discretos son más apropiados para evaluar la heterogeneidad. Sin embargo, sólo 6 EES aplicaron este tipo de modelo, lo que debería ser considerado en estudios posteriores para la obesidad y otros temas de importancia para la salud pública basada en la evidencia.

En el mismo sentido, la mayoría de las evaluaciones económicas completas utilizaron cadenas de Márkov y árboles de decisión, los cuales son limitados para trastornos crónicos como la obesidad ya que no captan cambios en los estados de salud en el tiempo ni entre subgrupos de pacientes y sus comorbilidades. Sin embargo, su uso en los estudios de esta revisión podría 
FIGURA 3. Cantidad de evaluaciones económicas y distribución geográfica

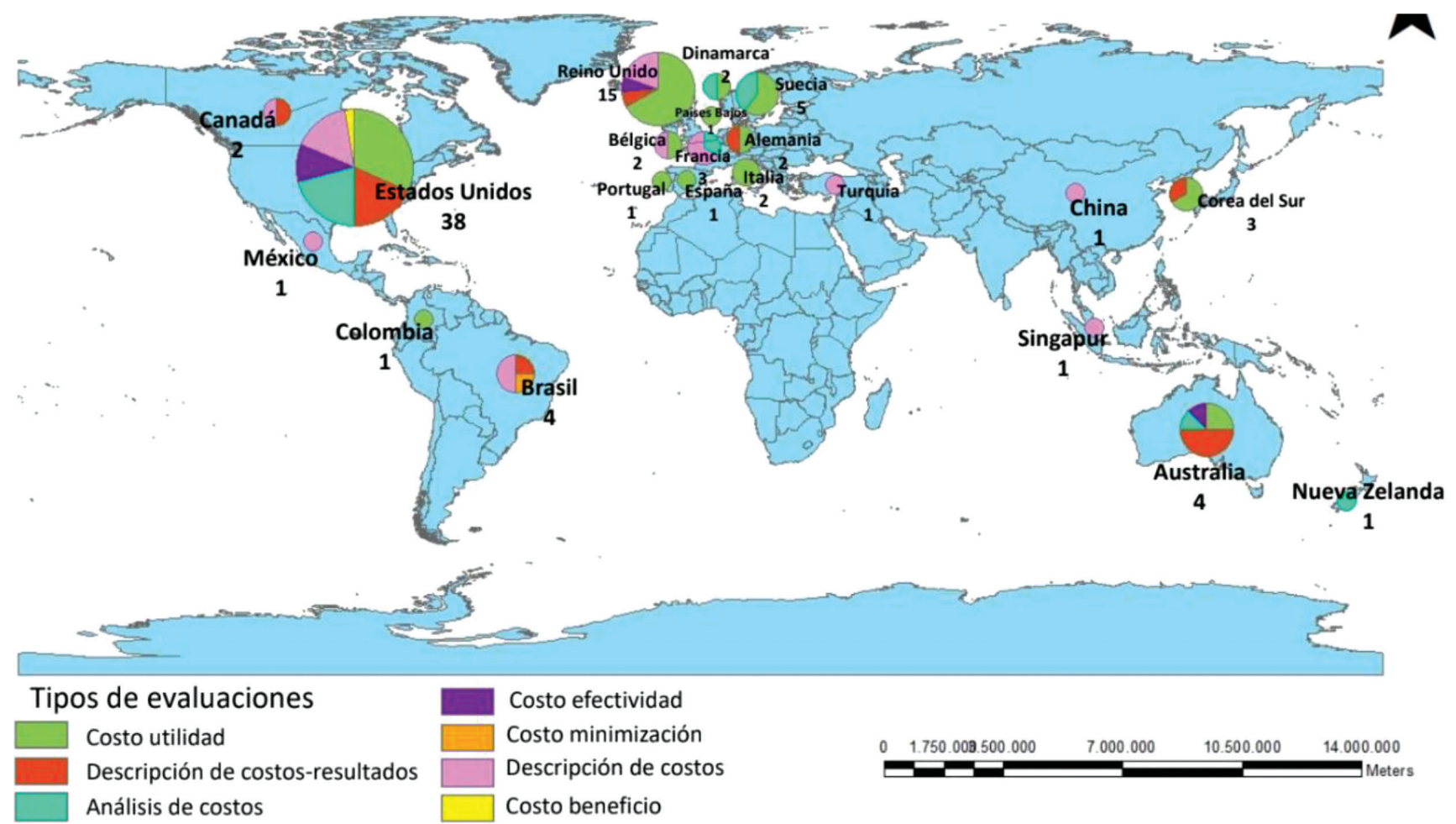

Fuente: Elaboración propia a partir de datos publicados.

TABLA 1. Caracterización de los estudios incluidos según pregunta PICOR (Población, Intervención, Comparador, Desenlace, Recursos)

\begin{tabular}{|c|c|c|c|c|c|c|}
\hline & Total & Descripción de costos & $\begin{array}{l}\text { Descripción de costos y } \\
\text { resultados }\end{array}$ & Análisis de costos & Costo-efectividad & Costo-utilidad \\
\hline \multicolumn{7}{|l|}{ Comorbilidades } \\
\hline Cardiovasculares & $58,9(56)$ & $66,7(10)$ & $56,3(9)$ & $52,9(9)$ & $57,1(4)$ & $61,5(24)$ \\
\hline Apnea del sueño & $13,7(13)$ & $40,0(6)$ & $0,0(0)$ & $23,5(4)$ & $0,0(0)$ & $7,7(3)$ \\
\hline Cáncer & $9,5(9)$ & $6,7(1)$ & $18,8(3)$ & $5,9(1)$ & $0,0(0)$ & $10,3(4)$ \\
\hline Quirúrgica & $55,8(53)$ & $73,3(11)$ & $18,8(3)$ & $64,7(11)$ & $42,9(3)$ & $61,5(24)$ \\
\hline Estilos de vida & $33,7(32)$ & $13,3(2)$ & $68,8(11)$ & $23,5(4)$ & $42,9(3)$ & $30,8(12)$ \\
\hline Farmacológica & $7,4(7)$ & $0,0(0)$ & $6,3(1)$ & $11,8(2)$ & $14,3(1)$ & $7,7(3)$ \\
\hline Convencional & $3,2(3)$ & $13,3(2)$ & $6,3(1)$ & $0,0(0)$ & $0,0(0)$ & $0,0(0)$ \\
\hline \multicolumn{7}{|l|}{ Comparador $^{\mathrm{a}}$} \\
\hline Convencional & $34,7(33)$ & $N A$ & NA & $17,6(3)$ & $57,1(4)$ & $66,7(26)$ \\
\hline Farmacológico & $1,1(1)$ & NA & NA & $0,0(0)$ & $0,0(0)$ & $2,6(1)$ \\
\hline \multicolumn{7}{|l|}{ Desenlace $^{\mathrm{a}}$} \\
\hline AVAC & $44,2(42)$ & NA & $18,8(3)$ & NA & NA & $100,0(39)$ \\
\hline Pérdida de peso & $14,7(14)$ & NA & $56,3(9)$ & NA & $71,4(5)$ & NA \\
\hline AVAD & $5,3(5)$ & NA & $18,8(3)$ & NA & $28,6(2)$ & NA \\
\hline Estancia hospitalaria & $1,1(1)$ & NA & $6,3(1)$ & NA & $0,0(0)$ & NA \\
\hline
\end{tabular}


TABLA 1. Caracterización de los estudios incluidos según pregunta PICOR (Población, Intervención, Comparador, Desenlace, Recursos) (continúa)

\begin{tabular}{|c|c|c|c|c|c|c|}
\hline & Total & Descripción de costos & $\begin{array}{l}\text { Descripción de costos y } \\
\text { resultados }\end{array}$ & Análisis de costos & Costo-efectividad & Costo-utilidad \\
\hline \multicolumn{7}{|l|}{$\begin{array}{l}\text { Recursos } \\
\text { (tipo de costo) }\end{array}$} \\
\hline Directos & $78,9(75)$ & $93,3(14)$ & $62,5(10)$ & $88,2(15)$ & $42,9(3)$ & $84,6(33)$ \\
\hline Indirectos & $20,0(19)$ & $6,7(1)$ & $37,5(6)$ & $11,8(2)$ & $57,1(4)$ & $15,4(6)$ \\
\hline \multicolumn{7}{|c|}{ Recursos (perspectiva) } \\
\hline Prestador & $34,7(33)$ & $60,0(9)$ & $31,3(5)$ & $41,2(7)$ & $71,4(5)$ & $17,9(7)$ \\
\hline Social & $16,8(16)$ & $13,3(2)$ & $31,3(5)$ & $11,8(2)$ & $14,3(1)$ & $12,8(5)$ \\
\hline
\end{tabular}

\%: proporción de columna (el denominador es cada tipo de evaluación económica).

a En $33 \%$ de los estudios no aplica esta característica; corresponde a las evaluaciones económicas descriptivas (costo, costos y resultados).
NA, no aplica; AVAD, años de vida ajustados por discapacidad; AVAC; años de vida ajustados por calidad. Fuente: Elaboración propia a partir de datos publicados.

TABLA 2. Costos totales y razones incrementales de costo-utilidad de las intervenciones identificadas en los estudios sistematizados

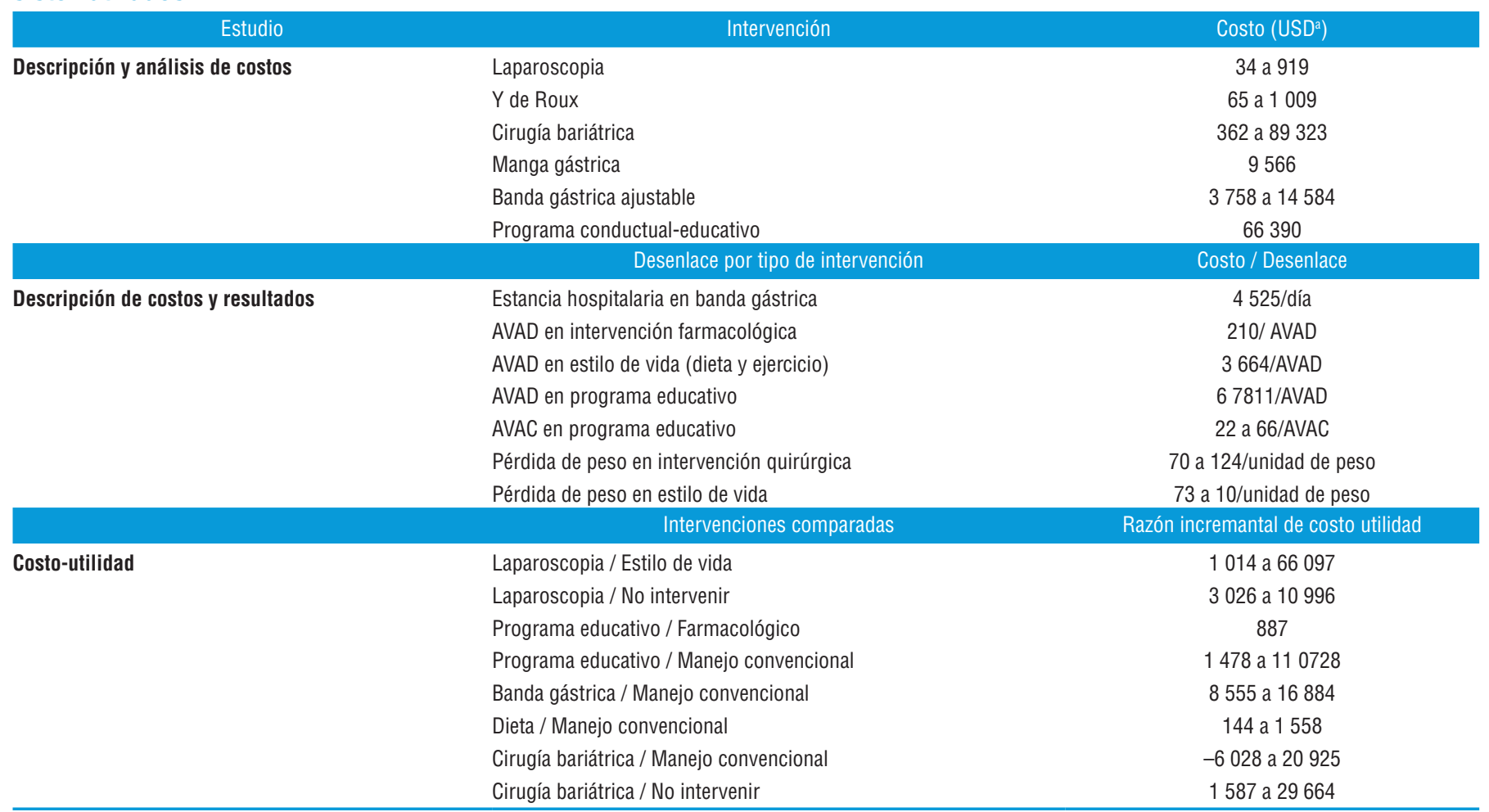

AVAD, años de vida ajustados por discapacidad; AVAC, años de vida ajustados por calidad.

a Costos en dólares estadounidenses de 2019 .

Fuente: Elaboración propia a partir de datos publicados.

justificarse por la escasa evidencia clínica y sanitaria de que se dispone para evaluar efectos en el largo plazo, al tiempo que esta situación reflejaría un obstáculo para generar evidencia de alta calidad en las EES ${ }^{18}$. Si bien la mayoría de las EES completas realizaron análisis de sensibilidad -determinantes para que los tomadores de decisiones conozcan el nivel de incertidumbre de los parámetros del modelo ${ }^{17-19}$-, se debe destacar la importancia de construir el modelo de manera rigurosa y coherente con las exigencias clínico-epidemiológicas.

Por otra parte, las EES consideraron todos los tipos de obesidad, aunque para futuras revisiones sería importante identificar cómo se afectan los resultados económicos según los tipos de obesidad. Algo similar sucede con el reporte de las comorbilidades; aunque se destaca la diabetes, se requiere una mejor estimación de los costos según el control de las enfermedades asociadas, lo que ayudaría a identificar los subgrupos donde las intervenciones maximizarían la eficiencia del uso de los recursos sanitarios ${ }^{17}$.

La mayoría de las intervenciones fueron quirúrgicas y carecieron de un comparador equiparable, ya que estos eran no intervenir, un tratamiento convencional poco definido, o carecían de un comparador, y es posible que este tipo de 
características del modelo defina los resultados de la EES. Las estrategias farmacológicas fueron poco evaluadas, lo que podría obedecer a que en la actualidad hay pocos fármacos aprobados para este uso y existe una evidencia débil sobre su efectividad clínica ${ }^{20}$.

En los desenlaces de salud se halló una alta variabilidad en cuanto al instrumento o la metodología de medición, por lo que es difícil comparar las distintas EES. El parámetro más usado y recomendado es los $\mathrm{AVAC}^{21}$ debido a que captan información relevante del curso biológico de la obesidad y el impacto de la intervención, pero la mayoría de los estudios realizaron la ponderación de calidad con datos de la literatura y la pérdida de peso, lo que excluye de la estimación dimensiones determinantes que no necesariamente están asociadas a la pérdida de peso y derivarían en una subvaloración de los resultados ${ }^{14}$.

Las EES relacionadas con los estilos de vida fueron parciales, la mayoría consideró medidas asociadas al peso y análisis de corto plazo; algunos autores refieren que este tipo de intervenciones suelen generar pequeñas pérdidas de peso y no mostrar suficientes rentabilidades a corto plazo $^{22-25}$.

La perspectiva más frecuente entre las evaluaciones económicas identificadas en la revisión sistemática fue la del prestador o tercer pagador; esto tiene implicaciones directas en las conclusiones dado que se podrían subestimar los costos al no incluir los gastos de bolsillo, el tiempo de los cuidadores y las pérdidas de productividad, que en el caso de la obesidad son muy altas $^{17-19}$. Además, se observó que en la mayoría de las EES la estimación de los costos fue derivada de la construcción y supuestos de los modelos, lo que podría sobrestimar algunos costos del comparador usado, por lo que los análisis incrementales deben interpretarse a la luz de dichos supuestos.

Las limitaciones del estudio incluyen la ausencia de registro del protocolo de revisión; potenciales sesgos de publicación por las restricciones de idioma de la búsqueda; la ausencia de síntesis cuantitativa o metaanálisis; y la imposibilidad de identificar las mejores intervenciones para la obesidad en adultos debido a la alta diversidad de los estudios en temas como el tipo de evaluación económica, costos incluidos, tipo de comparadores y desenlaces evaluados, lo cual es una características propia de las revisiones bajo el enfoque amplio de Cochrane. A pesar de las limitaciones, este tipo de revisiones permite una aproximación empírica de síntesis de la evidencia científica en un campo, actualizar las recomendaciones actuales, analizar el potencial de generalización de los estudios publicados, definir hipótesis con evidencia sólida o insuficiente e identificar áreas de investigación posterior.

Se concluye que los estudios microeconómicos para el tratamiento de la obesidad se han incrementado en los últimos años; sin embargo, persisten vacíos como la baja aplicación de intervenciones no quirúrgicas, evaluaciones parciales o de costo-efectividad y análisis desde la perspectiva social; estos constituyen ejes importantes para las prácticas en salud basadas en evidencia y una toma de decisiones que relacione los beneficios en salud con los costos implicados. La síntesis de evaluaciones económicas constituye un campo de gran importancia para la salud pública contemporánea.

Contribución de los autores. JMSG y MIBM concibieron el estudio, recolectaron la información, planificaron el desarrollo, analizaron los datos y escribieron el manuscrito. JACA concibió el estudio, analizó los datos y escribió el manuscrito. Todos los autores interpretaron los resultados, revisaron el manuscrito y aprobaron la versión final.

\section{Conflictos de intereses. Ninguno.}

Declaración. Las opiniones expresadas en este manuscrito son únicamente responsabilidad de los autores y no reflejan necesariamente las de la Revista Panamericana de Salud Pública o la Organización Panamericana de la Salud.

\section{REFERENCIAS}

1. Butland B, Jebb S, Kopelman P, McPherson K, Thomas S, Mardell J, et al. Tackling obesities: future choices-Project report. UK: Government Office for Science; 2007 [citado el 5 de mayo de 2020]. Disponible en: http://www.bis.gov.uk/assets/foresight/docs/ obesity/17.pdf.

2. Organización para la Cooperación y el Desarrollo Económicos (OCDE). Actualización de obesidad. OCDE; 2017 [citado el 5 de mayo de 2020]. Disponible en: http://www.oecd.org/els/health -systems/obesity-update.htm.

3. Organización Mundial de la Salud. 10 datos sobre la obesidad. Ginebra: OMS; 2017 [citado el 5 de mayo de 2020]. Disponible en: http://www.who.int/features/factfiles/obesity/es/.

4. Asamblea General de las Naciones Unidas. Declaración política de la reunión de alto nivel de la asamblea general sobre la prevención y el control de las enfermedades no transmisibles. New York: ONU; 2016 [citado el 5 de mayo de 2020]. Disponible en: http://undocs. org/es/A/66/.

5. República de Colombia, Ministerio de Salud y Protección Social. Resolución Número 2465 de 2016. Por la cual se adoptan los indicadores antropométricos, patrones de referencia y puntos de corte para la clasificación antropométrica del estado nutricional de niñas, niños y adolescentes menores de 18 años de edad, adultos de 18 a 64 años de edad y gestantes adultas y se dictan otras disposiciones. Bogotá: MSPS; 2016 [citado el 5 de mayo de
2020]. Disponible en: https: / / www.icbf.gov.co/sites/default/files / resolucion_no._2465_del_14_de_junio_de_2016.pdf.

6. Organización para la Cooperación y el Desarrollo Económicos. The Heavy Burden of Obesity: The Economics of Prevention. Paris: OECD Health Policy Studies, OECD Publishing; 2019. https:/ /doi. org $/ 10.1787 / 67450$ d67-en.

7. Moreno M. Definición y clasificación de la obesidad. Rev Médica Clínica Las Condes. 2012;2(23):124-8.

8. Jefferson T, Demicheli V, Vale L. Quality of systematic reviews of economic evaluations in health care. JAMA. 2002; 287(21):2809-12.

9. Zanganeh, M., Adab, P., Li, B. et al. Protocol for a systematic review of methods and cost-effectiveness findings of economic evaluations of obesity prevention and/or treatment interventions in children and adolescents. Syst Rev. 2018;7:54. https://doi.org/10.1186/ s13643-018-0718-5

10. Cardona-Arias J, Higuita-Gutiérrez L, Ríos Osorio L. Revisiones sistemáticas de la literatura científica: la investigación teórica como principio para el desarrollo de la ciencia básica y aplicada Bogotá: Ediciones Universidad Cooperativa de Colombia; 2016.

11. Higgins J, Green S. Manual Cochrane de revisiones sistemáticas de intervenciones. Traducción a cargo del Centro Cochrane Iberoamericano. [Internet] 2011; [citado el 5 de mayo de 2020]. Disponible en: www.cochrane-handbook.org. 
12. Drummond M. Métodos para la evaluación económica de los programas de asistencia sanitaria. Madrid: Díaz de Santos; 2001.

13. Organización para la Cooperación y el Desarrollo Económicos. Inflation (CPI). OECD; 2020; [citado el 5 de mayo de 2020]. Disponible en: https://data.oecd.org/price/inflation-cpi.htm

14. Jia H, Lubetkin E. The impact of obesity on health-related quality-of-life in the general adult US population. J Public Health. 2005;27(2):156-64

15. Hutubessy R, Chisholm D, Tan-Torres T y OMS-CHOICE. Análisis de costo-efectividad generalizado para el establecimiento de prioridades del sector salud en el ámbito nacional. Washington: OPS; 2003 [citado el 5 de septiembre de 2020]. Disponible en: https:/ / www.paho.org/ per/images/stories/FtPage/2011/20111207_costo.pdf?ua=1.

16. Instituto de Evaluación Tecnológica en Salud. Manual para la elaboración de evaluaciones económicas en salud. Bogotá: IETS; 2014.

17. Alsumali A, Al-Hawag A, Samnaliev M, Eguale T. Systematic assessment of decision analytic models for the cost-effectiveness of bariatric surgery for morbid obesity. Surg Obes Relat Dis. 2018;14(7):1041-1059.

18. Wang B, Furnback W. Modelling the long-term outcomes of bariatric surgery: a review of cost-effectiveness studies. Best Pract Res Clin Gastroenterol. 2013; 27(6):987-995.

19. Zabatiero J. Effectiveness of interventions aiming at reducing sedentary behaviour in a non-surgical population with overweight or obesity: A systematic review and meta-analysis. Obes Res Clin Pract. 2019;13(2):115-128.

20. Londoño-Lemos M. Aproximaciones farmacológicas para el tratamiento de la obesidad / Pharmacological approaches to the treatment of obesity. Rev Colomb Cienc Quím-Farm. 2017;1:84
21. Jia $H$, Lubetkin E. Obesity-related quality-adjusted life years lost in the US from 1993 to 2008. Am J Prev Med. 2010;39(3):220-227.

22. Loveman E, Frampton GK, Shepherd J, et al. The clinical effectiveness and cost-effectiveness of long-term weight management schemes for adults: a systematic review. 2011. In: NIHR Health Technology Assessment programme: Executive Summaries. Southampton (UK): NIHR Journals Library; 2003; [citado el 5 de mayo de 2020]. Disponible en https://www.ncbi.nlm.nih.gov/books/ NBK56859/

23. Estabrooks PA, Wilson KE, McGuire TJ, Harden SM, Ramalingam N, Schoepke L, et al. A Quasi-Experiment to Assess the Impact of a Scalable, Community-Based Weight Loss Program: Combining Reach, Effectiveness, and Cost. J Gen Intern Med. 2017;32(S1):24-31.

24. Cobiac L, Vos T, Veerman L. Cost-effectiveness of Weight Watchers and the Lighten Up to a Healthy Lifestyle program. Aust N Z J Public Health. 2010;34(3):240-7.

25. Forster M, Veerman JL, Barendregt JJ, Vos T. Cost-effectiveness of diet and exercise interventions to reduce overweight and obesity. Int J Obes. 2011;35(8):1071-8.

Manuscrito recibido el 26 de junio de 2020. Aceptado para su publicación, tras revisión, el 8 de septiembre de 2020.

\section{Systematic review of health economic evaluations for the treatment of obesity in adults, 2009-2019}

ABSTRACT Objective. To analyze health economic evaluations for the treatment of obesity in adults published globally between 2009-2019.

Methods. Systematic review applying Cochrane, PRISMA and CHEERS guidelines for health economics studies. The selection and information extraction protocol assured the completeness, reproducibility and methodological quality. Qualitative synthesis was carried out by means of frequency of type of economic evaluation.

Results. We screened 4493 articles and only 95 met the protocol criteria, most of them from the United States $(n=38)$, mainly with cost-utility studies $(n=39)$ and cost descriptions $(n=18)$. People with multiple comorbidities were included, the most common of which was diabetes. The most evaluated interventions were surgical $(n=53)$, and the most frequent outcomes were weight reduction and quality adjusted life years. The most frequent evaluation perspective was that of the third payer $(n=46)$; most common timeline was the short term for lifestyle interventions and the long term for surgical ones.

Conclusion. Microeconomic studies for the treatment of obesity have increased in recent years; however, gaps remain such as the low application of non-surgical interventions, partial or cost-effectiveness evaluations, and analyses from the social perspective. These represent important aspects for evidence-based health practices and decision making relating health benefits to the costs involved.

Keywords Obesity; communicable disease control; health economics; systematic review; adult. 